$1352 \mathrm{ng} / \mathrm{mL}$ after allegedly increasing his dose to $700 \mathrm{ug} / \mathrm{day}$. Extrapolating the $400 \mathrm{ug} /$ day data to $700 \mathrm{ug} / \mathrm{day}$, and assuming an increase from twice-daily to three-times daily dosing, the Salbutamol Passport prediction is $1050 \mathrm{ng} / \mathrm{mL}$ (95\%PI: 802-1375). Since the observed test falls within the 95\% prediction interval, the result would not constitute an AAF. This conclusion is unchanged after adjustment of salbutamol levels to a urine specific gravity of 1.020 .

\section{DIETARY PRACTICES OF PHYSICALLY ACTIVE 9-12-YEAR OLD CHILDREN, PHYSICAL ACTIVITY AND NUTRITIONAL KNOWLEDGE OF THEIR PARENTS}

EC Wauchope. Department of Sociology (Sport and Exercise Sciences), Durham University, UK

\subsection{6/bjsports-2018-ISSSMC2018.9}

Healthy eating through a balanced diet is fundamental to good health. Active children are of interest given the energy demands of preparing for athletic performance or competition alongside normal growth and development. There is minimal research into the requirements of preadolescent athletes. It is unknown whether findings and recommendations in adult athletes can be extrapolated to their junior counterparts. Active 9-12 year old children $(n=18)$ from the North East of England completed three-day estimated weight food diaries, like the National Diet and Nutrition Survey (2016). Secondary outcomes determined self-reported physical activity level using the Physical Activity Questionnaire for Children, and parental scores of the General Nutrition Knowledge Questionnaire. Daily averages of energy, fibre, macronutrients (protein, carbohydrates and fat) and key micronutrients (Vitamin C, Vitamin $\mathrm{D}$, iron, calcium and sodium) were analysed using McCance and Widdowson's Composition of Foods (2015). To contextualise the nutrition findings, data were compared to national recommendations and the National Diet and Nutrition Survey (2016). Energy intake (7.2 MJ/day) was higher than for children nationally but lower than recommended. This was principally driven by low intake of carbohydrates $(234 \mathrm{~g} /$ day $)$ and fat $(62 \mathrm{~g} /$ day $)$. Intake of protein was comparatively high $(69 \mathrm{~g} /$ day) and total fibre $(9 \mathrm{~g} /$ day $)$ low. Intake of Vitamin C (112 mg/day), iron (13 mg/day) and calcium (732 mg/day) was sufficient and Vitamin D $(2.5 \mu \mathrm{g} /$ day $)$ low. Secondary outcomes analysed associations between daily nutritional values and child physical activity and parent nutritional knowledge scores using the Pearson correlation co-efficient. There were no relationships $(p>0.05)$ between energy intake and either of these variables. Findings suggested that active 9-12 year old children consume more energy than indicated by UK datasets, but consume less energy than national recommendations for gender and age. Principally this was driven by low intake of carbohydrate and fat. No significant relationships were apparent between energy intake and physical activity level, or energy intake and parent nutrition knowledge score. These findings are important to better-understand and support nutritional needs of active children.

\section{COMPARING THREE ANALYSIS METHODS FOR KINEMATIC DATA EVALUATION}

$\mathrm{S}$ Alizadeh, K Mattes. Department of Movement and Training Sciences, University of Hamburg, Hamburg, Germany

\subsection{6/bjsports-2018-ISSSMC2018.10}

The purpose of this study was to compare three different methods of data analysis: conventional, Statistical Non-Parametric Mapping (SnPM) and Functional Data Analysis (FDA) for a series of kinematic data acquired from soccer players from their dominant and non-dominant leg at the late swing phase while sprinting. Kinematic data was acquired via Vicon motion analysis system at $200 \mathrm{~Hz}$. For conventional computation, local maximum, mean and minimum values were extracted for further analysis. A two-tailed dependent t-test was implemented for statistical analysis for the conventional method. A two-tailed SnPM and a one-tailed FDA permutation test was performed on the same data $(\alpha=0.05)$. All the trials where filtered using zero-lag 4th order Butterworth filter. The cutoff frequency $(13 \mathrm{~Hz})$ was calculated using the optimum cutoff frequency formula. Late-swing phase was defined as the moment where the knee started to extend from its flexed position in the 2 nd half of swing phase till foot contact. The conventional results show there was a difference in the mean knee angle in the sagittal plane between the dominant $\left(\theta^{\circ}=73.0\right)$ and non-dominant leg $\left(\theta^{\circ}=74.7\right)$ $(\mathrm{p}=0.04)$. Both SnPM (\% late swing phase found significant $=28.9 \%-86.1 \%, \quad \mathrm{t}$-value $=2.52, \mathrm{p}<0.001)$ and FDA $(\%$ late swing phase found significant $=28.1 \%-87.7 \%$, tvalue $=2.47-2.55, \mathrm{p}<0.001$ ) exhibited a significant difference between the knee angle of the dominant and non-dominant leg at the late swing phase. However, the SnPM demonstrated reliability in producing result while FDA presented a fluctuation, although minor, in the calculation of $p$ value. SnPM would produce more reliable and comprehensive result in comparison to FDA and conventional methods. SnPM enables scientist to identify deviation in movement pattern in soccer players which is not limited to a single point in time and can identify deficiency in movement pattern which may hinder performance or lead to injury. 